\title{
INSUFICIENNCIA RENAL AGUDA ASSOCIADA AO USO DE ACICLOVIR ENDOVENOSO
}

\author{
Leonardo R. Pacheco, Helder M. E. Tavares, Miguel Moysés Neto, Marcio Dantas, luís Sérgio D’Oliveira Rocha, Karina Mota Ribeiro, \\ José Fernando de Castro Figueiredo* \\ Trabalho realizado no Departamento de Clínica Médica da Faculdade de Medicina de Ribeirão Preto da Universidade de São Paulo, \\ Ribeirão Preto, SP.
}

\author{
*Correspondência: \\ Divisão de Moléstias \\ Infecciosas e Tropicais. \\ Departamento de Clínica \\ Médica. Faculdade de \\ Medicina de Ribeirão Preto \\ da Universidade de São \\ Paulo. Av. Bandeirantes, \\ 3.900, Bairro Monte Alegre, \\ Ribeirão Preto, SP, \\ Cep 14049-900 \\ Tel: (16) 602-2468, \\ Fax: (16) 633-6695. \\ ifcfigue@fmrp.usp.br
}

\begin{abstract}
RESUMO
OBJEtıvo. Avaliar a incidência e evolução da IRA em pacientes que utilizaram aciclovir por via intravenosa.

Métodos. Foram revisados, durante um período de sete meses consecutivos, os prontuários médicos de pacientes acima de 13 anos de idade que usaram aciclovir endovenoso (EV) por cinco dias ou mais. A IRA foi considerada quando a creatinina sérica, previamente normal, aumentava acima de $2 \mathrm{mg} / \mathrm{dl}$. Foi analisado o tipo de tratamento instituído nos casos de IRA e sua evolução.

REsULTADOS. Oitenta e cinco pacientes receberam aciclovir por via endovenosa durante o período estudado. Foram incluídos no estudo 4 I pacientes. A IRA desenvolveu-se em 8 dos 4 I pacientes estudados (19,5\%). 0 tempo médio para o início do aumento dos níveis séricos da creatinina, após o início do uso da droga, foi de 4,2 dias, com o pico dos níveis da creatinina sérica aparecendo entre 3 a I4 dias (média 7,I dias). A recuperação da função renal, avaliada pela queda dos níveis da creatinina, variou de I a 7 dias ( média de 3,6 dias). A resolução da IRA ocorreu após medidas gerais de hidratação, aumento do tempo de infusão e ajuste da dose do aciclovir.

Conclusä̃o. O aciclovir provocou IRA em 19,5\% dos pacientes, que evolúram bem em todos os casos observados, com retorno da função renal pré-tratamento após medidas de hidrataçãao, reajuste da dose e aumento do tempo de infusão. Não houve necessidade de hemodialise em nenhum paciente. A droga apresenta segurança de uso, desde que cuidados sejam implementados durante sua administração.
\end{abstract}

UnItermos: Insuficiência renal aguda. Aciclovir.

\section{INTRODUÇÃO}

O aciclovir é uma droga antiviral, com baixa toxicidade para células humanas'. É um inibidor altamente efetivo da replicação de herpes simplex I e II e do vírus varicela Zoster ${ }^{\prime, 2}$ e é amplamente utilizado em pacientes imunossuprimidos, em particular os portadores de síndrome da imunodeficiência adquirida (SIDA) e naqueles submetidos a transplante, tanto de medula óssea, quanto de órgãos sólidos, incluindo o rim. Em pacientes imunocompetentes, é usado no tratamento de herpes genital e infecções herpéticas do sistema nervoso central?2.

O ótimo uso desta droga requer o conhecimento de sua farmacologia e de seus potenciais efeitos colaterais. $\mathrm{O}$ aciclovir pode apresentar neuro e nefrotoxicidade por mecanismos ainda não totalmente esclarecidos ${ }^{3,4,5,6}$. É rapidamente excretado na urina por filtração glomerular e secreção tubular e $60 \%$ a $90 \%$ da droga é excretada inalterada na urina, alcançando altas concentrações intratubulares ${ }^{2.7}$. Sua solubilidade é relativamente baixa e pode desencadear quadro de insuficiência renal aguda (IRA) após doses elevadas ou infusões endovenosas rápidas 2,3,4,5.

O objetivo do presente trabalho foi avaliar a incidência e evolução da IRA em pacientes que utilizaram aciclovir por via intravenosa e identificar os possíveis fatores de risco associados.

\section{Métodos}

Foram revisados os prontuários médicos de todos os pacientes do Hospital das Clínicas da Faculdade de Medicina de Ribeirão Preto da
Universidade de São Paulo (HCFMRP-USP) que usaram o aciclovir endovenoso (EV) por cinco dias ou mais, no período de março a setembro de 2002. Foram avaliados os seguintes dados: idade do paciente, doença de base, indicação do uso da droga, tempo de uso e a dose total recebida, drogas associadas, evolução da creatinina em mg/ dL. Foram excluídos os pacientes com idade inferior a I 3 anos de idade.

A IRA foi considerada quando a creatinina sérica, previamente normal (os valores de referência do método utilizado no HCFMRPUSP se situam entre 0,7 e I, $5 \mathrm{mg} / \mathrm{dL}$ ), aumentava acima de $2 \mathrm{mg} / \mathrm{dL}$ ou, em casos de insuficiência renal prexistente, se elevava pelo menos $2 \mathrm{mg} / \mathrm{dL}$ acima do nível pré-tratamento. Além disso, foram analisados o tipo de tratamento instituído nos casos de IRA e sua evolução.

Os dados foram analisados empregando-se os testes de Mann Whitney e $\chi 2$, com nível de significância de 5\%. O trabalho foi realizado com a aprovação do Comitê de Ética em Pesquisa do Hospital das Clínicas de Ribeirão Preto-USP (Processo nº 4306/2004).

\section{Resultados}

Oitenta e cinco pacientes receberam aciclovir por via endovenosa durante o período de estudo e um total de 49 pacientes fizeram uso da droga por cinco dias ou mais. Desse total, oito pacientes tinham idade abaixo de 13 anos e foram excluídos do estudo, sendo avaliados, no final, 4 I pacientes. As características gerais destes pacientes e as indicações do uso do aciclovir encontram-se na Tabela I. 
Pacheco LR et al.

A incidência de IRA após exposição ao aciclovir foi de 19,5\% (8/4I). A mediana (percentis 25\%; e $75 \%$ ) da creatinina sérica prévia dos pacientes do grupo que desenvolveu IRA foi de $0,95 \mathrm{mg} / \mathrm{dL}(0,87 ; \mathrm{I}, \mathrm{I}$ ) atingindo um pico de $3, \mathrm{Img} / \mathrm{dL}(2,8 ; 3,7)$. O tempo médio para o início do aumento dos níveis séricos da creatinina, após o início do uso da droga, foi de 4,2 dias (variando de I a 7 dias), com o pico dos níveis da creatinina sérica ocorrendo entre 3 a 14 dias (média 7,1 dias). O tempo para a recuperação da função renal, avaliada pela queda dos níveis da creatinina, variou de I a 7 dias (média de 3,6 dias) (Tabela 2). Todos os pacientes evoluíram com IRA não oligúrica. A resolução da IRA ocorreu após medidas gerais de hidratação, aumento do tempo de infusão e ajuste da dose do aciclovir, além da retirada de medicações concomitantes, consideradas nefrotóxicas (Tabela 3). Dois pacientes, em razão de complicação da doença de base, evoluíram para o óbito, mesmo após a melhora da função renal.

Trinta e seis pacientes fizeram uso concomitante de outras drogas potencialmete nefrotóxicas, porém este fator não aumentou a probabilidade de desenvolver IRA. Da mesma forma, os níveis prévios de creatinina sérica não foram diferentes quando comparamos os pacientes dos dois grupos, com e sem IRA. Por outro lado, encontraram-se diferenças estatísticas significantes $(p<0,05)$ entre os que desenvolveram IRA e o grupo que não desenvolveu para a idade do paciente e dose total do aciclovir (Tabelas 3 e 4).

\section{Discussão}

Um dos primeiros relatos sobre alterações da função renal em pacientes que receberam aciclovir por via intravenosa mostrou que, de 354 pacientes, 58 (16,3\%) apresentaram aumentos dos níveis séricos de creatinina ${ }^{3}$. Essas alterações ocorreram após injeção em bolus de altas doses da droga. Em outro estudo, quatro pacientes $(14,2 \%)$ de 28 tratados com doses elevadas da droga apresentaram quadro de IRA, mesmo após infusão lenta do medicamento, em uma hora 5 . Em outro estudo, Perazella ${ }^{8}$ relata ocorrência de IRA em $48 \%$ dos pacientes que receberam a droga.

$\mathrm{Na}$ presente casuística, a IRA desenvolveu-se em oito dos 4I pacientes (19,5\%) estudados. O tempo médio para o desenvolvimento da IRA foi de 4,2 dias e o pico do aumento da creatinina sérica ocorreu em média 7,I dias após o início da droga. Nos oito casos que apresentaram aumento da creatinina sérica, a melhora da função renal ocorreu após o emprego de medidas conservadoras (reposição volêmica, ajuste da dose do aciclovir e/ou de outras drogas, redução da velocidade de infusão do aciclovir). A melhora se deu em média 3,6 dias depois, sem que houvesse a necessidade de suspensão do aciclovir, na maioria dos casos. Em apenas um paciente houve necessidade de suspensão do medicamento, por dois dias, com a sua re-introdução posterior, em doses menores. De fato, a literatura aponta que a maioria dos pacientes apresenta melhora da IRA após suspensão ou diminuição da droga, bem como após a hidratação adequada dos mesmos ${ }^{3,5}$. Em alguns casos, pode haver indicação de hemodiálise, uma vez que este procedimento pode remover eficientemente o aciclovir da circulação, especialmente quando há associação com neurotoxicidade ${ }^{4,6}$.

O mecanismo de lesão renal pelo aciclovir é ainda controverso. A excreção urinária deste medicamento ocorre por filtração e secreção

\begin{tabular}{lc}
\hline \multicolumn{2}{c}{$\begin{array}{c}\text { Tabela I - Características gerais dos } \\
\text { pacientes e indicações de uso do aciclovir }\end{array}$} \\
\hline Variável & $\mathbf{n}(\%)$ \\
\cline { 2 - 2 } Sexo (masculino) & $26(63,4)$ \\
Mediana da idade (anos) & $31(24 ; 49)^{*}$ \\
Doença de Base: & \\
$\quad$ SIDA & $10(24,4)$ \\
Doença hematológica & $\mid 7(41,4)$ \\
Transplante renal & $04(9,7)$ \\
$\quad$ Outros & $10(24,3)$ \\
Indicações de uso do aciclovir & \\
Profilaxia em transplante de medula óssea & $7(17,0)$ \\
Infecções por vírus herpes simplex em: & \\
$\quad$ Sistemanervoso central & $8(19,5)$ \\
Pele e mucosas & $\mid 2(29,3)$ \\
Infecções por vírus varicella Zoster & $\mid 3(31,7)$ \\
Profilaxia em paciente com neutropenia & $\mid(2,4)$ \\
\hline * (percentil 25\%; percentil 75\%) &
\end{tabular}

* (percentil 25\%; percentil 75\%)

\begin{tabular}{lccc}
\hline \multicolumn{5}{c}{$\begin{array}{c}\text { Tabela 2 } \\
\text { creatinina sérica nos nos pacientes que apresentaram IRA }\end{array}$} \\
\hline Paciente & $\begin{array}{c}\text { Iń́cio } \\
\text { (dias) }\end{array}$ & $\begin{array}{c}\text { Pico } \\
\text { (dias) }\end{array}$ & $\begin{array}{c}\text { Normalização } \\
\text { (dias) }\end{array}$ \\
\hline & $\frac{1}{7}$ & 9 & 4 \\
2 & 6 & 5 & 7 \\
3 & 4 & 4 & 2 \\
4 & 3 & 8 & 4 \\
5 & 1 & 14 & 4 \\
6 & 7 & 7 & 5 \\
7 & 4 & 3 & 2 \\
8 & 2 & 7,1 & 3,6 \\
Média & 4,2 & & \\
\hline
\end{tabular}

tubular1,3,5 e a excreção urinária da droga não metabolizada corresponde a aproximadamente $62 \%$ a $91 \%$ da sua eliminação 13,5 . Como o aciclovir é relativamente insolúvel na urina, a rápida injeção intravenosa pode contribuir para a precipitação intratubular de cristais do medicamento 8,9 . Existem evidências de que a deposição de cristais intratubulares, com conseqüente obstrução, seja o principal fator responsável pelo desenvolvimento da IRA ${ }^{3,5,8,9}$. Além disso, o aciclovir pode, também, induzir a redução da taxa de filtração glomerular. Essa redução teria como substrato alguns fenômenos hemodinâmicos, tais como o aumento na resistência vascular total e da resistência da arteríola aferente, a redução na taxa de filtração glomerular e do fluxo plasmático renal e glomerular, além da alteração no coeficiente de permeabilidade glomerular ${ }^{10}$. Além dessas alterações na taxa de filtração glomerular, foi demonstrado que o aciclovir pode provocar lesões tubulares levando a poliúria e hipofosfatemia ${ }^{11,12}$. Em casos mais raros, a nefrite túbulo-intersticial aguda e a necrose tubular aguda poderiam também ser as causas da insuficiência renal ${ }^{13,14}$. Nos casos observados no presente trabalho, a rápida resolução da IRA após as medidas 


\begin{tabular}{|c|c|c|c|c|}
\hline \multicolumn{5}{|c|}{ Tabela 3 - Medidas empregadas para a reversão da IRA } \\
\hline Paciente & Hidratação & $\begin{array}{l}\text { Redução da dose } \\
\text { do aciclovir }\end{array}$ & $\begin{array}{l}\text { Aumento do tempo } \\
\text { de infusão do aciclovir }\end{array}$ & $\begin{array}{l}\text { Suspensão/ajuste de } \\
\text { outras drogas }\end{array}$ \\
\hline | & Sim & Sim & & SuspensãolECA \\
\hline 2 & Sim & Sim & Sim & \\
\hline 3 & Sim & Sim & & \\
\hline 4 & Sim & $\operatorname{Sim}$ & & \\
\hline 5 & Sim & $\begin{array}{l}\text { Suspensão temporária do } \\
\text { aciclovir e posterior } \\
\text { redução da dose }\end{array}$ & & $\begin{array}{c}\text { Ajuste das doses de } \\
\text { anfotericina B, imipenem, } \\
\text { vancomicina e amicacina }\end{array}$ \\
\hline 6 & Sim & $\operatorname{Sim}$ & & \\
\hline 7 & Sim & Sim & & \\
\hline 8 & Sim & Sim & & \\
\hline
\end{tabular}

IECA: inibidor da enzima conversora de angiotensina I

Tabela 4 - Correlação entre alguns parâmetros clínicos e laboratoriais de
pacientes que receberam o aciclovir e o desenvolvimento de IRA

Dados expressos em mediana (percentil 25\%; percentil 75\%)

*p $>0,05 ;$ ** $p<0,05$ (Mann Whitney)

implementadas tornou pouco provável essas duas últimas causas.

A utilização concomitante de drogas nefrotóxicas poderia, também, predispor os pacientes ao desenvolvimento de IRA. Nos casos observados, no entanto, não houve essa correlação. Segundo a literatura, a nefrotoxicidade pelo aciclovir pode ser facilitada pela presença de lesão renal prévia, de estados de baixa perfusão renal, e por níveis séricos elevados de aciclovir, acima de $20 \mu g / \mathrm{ml}$, associados à infusão endovenosa rápida da droga 3,5,8,9. No presente trabalho, a função renal prévia foi semelhante nos dois grupos e não havia pacientes com estados indicativos de baixa perfusão renal. Alguns fatores predisponentes identificados foram a idade do paciente e a dose total de aciclovir recebida. Assim, os pacientes que utilizaram doses maiores de aciclovir poderiam ter tido mais chances de terem recebido altas doses do aciclovir em curto espaço de tempo e, provavelmente, a função renal teria se alterado pela infusão rápida da droga.

A alteração da função renal pelo aciclovir pode ser evitada, bem como a alteração hemodinâmica que se segue à rápida infusão por bolus. A infusão lenta de aciclovir, em pelo menos duas horas, e a sua maior diluição, parecem ser suficientes para evitar a IRA. Recomendase que a droga seja diluída em uma proporção de $500 \mathrm{mg}$ em $100 \mathrm{ml}$ ou I g em $200 \mathrm{ml}$ de soro glicosado $5 \%$, soro fisiológico $0,9 \%$ ou $0,45 \%$. Além disso, a manutenção de um fluxo de diurese em torno de 100 a I $50 \mathrm{ml} /$ hora, previamente à administração da droga, pode ter um efeito benéfico. Portanto, em alguns casos, a prescrição de diuréticos pode propiciar altos fluxos urinários desde que seja realizada hidratação adequada além da monitorização dos eletrólitos séricos ${ }^{8,9}$.

\section{Conclusão}

É importante ressaltar que, devido à excreção renal de aciclovir, a dose deve ser corrigida quando há insuficiência renal prévia para evitar especialmente 0 aparecimento de neurotoxicidade ${ }^{2,7}$. Em conclusão, na presente casuística, o aciclovir provocou IRA em 19,5\% dos pacientes, evoluindo bem em todos os casos observados, com retorno da função renal pré-tratamento após medidas de hidrataçãa, reajuste da dose e diminuição do tempo de infusão do aciclovir. Neste estudo retrospectivo, os achados corroboram dados da literatura de que a IRA nesses casos é reversível, com raros casos de seqüelas nefrológicas. Não houve necessidade de hemodiálise em nenhum paciente. Assim, a droga apresenta seguranç̧a de uso, desde que alguns cuidados sejam implementados, como acima descrito. Todavia, faz-se necessário o acompanhamento da função renal em todos os pacientes que utilizam altas doses de aciclovir por via endovenosa.

\section{Conflito de interesse: não há.}

\section{SUMMARY}

ACUTE ReNAL faILURE RELATED to INTRAVENOUS ACYCLOVIR

OBIECTIVE. The aim of this study was to evaluate the incidence and outcome of acute renal failure (ARF) in patients submitted to intravenous (IV) acyclovir treatment.

METHODS. All patients over 13 years of age that used intravenous acyclovirfor 5 or more days were retrospectively analyzed. When serum creatinine levels, previously in the normal range, increased above $2 \mathrm{mg} / \mathrm{dl}$, the case was considered an ARF. Treatment and outcome of patients that developedARF were analyzed.

RESULTS. Eighty-five patients received IV acyclovir during the study period. Forty-one patients were included in the study. ARF developed in 8 out of 4 I patients (19.5\%). In the ARF cases, after beginning of treatment, the average time for increase of the serum creatinine levels was 4.2 days. Creatinine levels reached their peak in a mean time of 7.1 days (ranging from 3 to 14 days). Recovery of the renal function, evaluated by decrease of the creatinine level, varied from I to 7 days (mean of 3.6 days). ARF had a good outcome with hydration, lengthening of drug infusion time and adjustment of the drug dosage. 
CONCLUSION. Acyclovir induced ARF in 19.5\% of the patients. All patients had a positive response with return to anormal renalfunction after hydration, lengthening of drug infusion time and adjustment of the drug dosage. None of the patients required treatment with hemodialysis. Acyclovir is a safe drug when administered with certain preventive measures. [Rev Assoc Med Bras 2005; 5 I (5): 275-8]

KEY WORDS: Acute renal failure. Acyclovir.

\section{RefERÊNCIAS}

I. Keeney RE, Kirk RE, Brigden D. Acyclovir tolerance in humans. Am J Med 1982;72: |A: | 76-8|

2. Laskin OL. Acyclovir pharmacology and clinical experience. Arch Intern Med 1984; | 44: | 24|-6

3. Brigden D, Rosling AE, Woods NC. Renal function after acyclovir intravenous infection. Am J Med 1982;73(Suppl IA): I 82-5.

4. Spiegal DM, Lau K. Acute renal failure and coma secondary to acyclovir therapy. JAMA 1986;255: I 882-3

5. Sawyer MH, Webb DE, Balow JE, Straus SE. Acyclovir-induced renal failure. Am J Med 1988;84:1067-71.

6. Krieble BF, Rudy DW, Glick MR, Clayman MD. Case report: acyclovir neurotoxicity and nephrotoxicity-the role for hemodialysis. Am J Med Sci 1993;305:36-9.
7. Blum MR, Liao SHT, Miranda P. Overview of acyclovir pharmacokinetic disposition in adults and children. Am J Med 1982;72: IA: I 86-92.

8. Perazella MA. Crystal-induced acute renal failure. Am J Med 1999; 106:459-64.

9. Perazella MA. Drug-induced renal failure: update on new medications and unique mechanisms of nephrotoxicity. Am J Med Sci 2003;325:349-62.

10. Dos Santos MFF, Santos OFP, Boim MA, Razvickas CV, Moura LAR, Ajzen $\mathrm{H}$, et al. Nephrotoxicity of acyclovir and ganciclovir in rats: evaluation of glomerular hemodynamics. J Am Soc Nephrol 1997;8:36 I-7.

11. Rashed A, Azadeh B, Romeh SHA. Acyclovir-induced acute tubulointerstitial nephritis. Nephron 1990;56:436-8.

12. Becker BN. Rapidly progressive acute renal failure due to acyclovir: case report and review of the literature. Am J Kidney Dis 1993;22:6 I I-5.

13. Campos SB, Seguro AC, Cesar KR, Rocha AS. Effects of acyclovir on renal function. Nephron 1992;62:74-9.

14. Andrade L, Rebouças NA, Seguro AC. Down-regulation of $\mathrm{Na}^{+}$ transporters and AQP2 is responsible for acyclovir-induced polyuria and hypophosphatemia. Kidney Int 2004;65: 175-83.

Artigo recebido: 17/05/04

Aceito para publicação: 16//2/04 